\title{
Reversible Posterior Leukoencephalopathy Syndrome Associated with Tacrolimus Therapy
}

Key words: reversible posterior leukoencephalopathy, tacrolimus, post-transplantation complication, magnetic resonance imaging
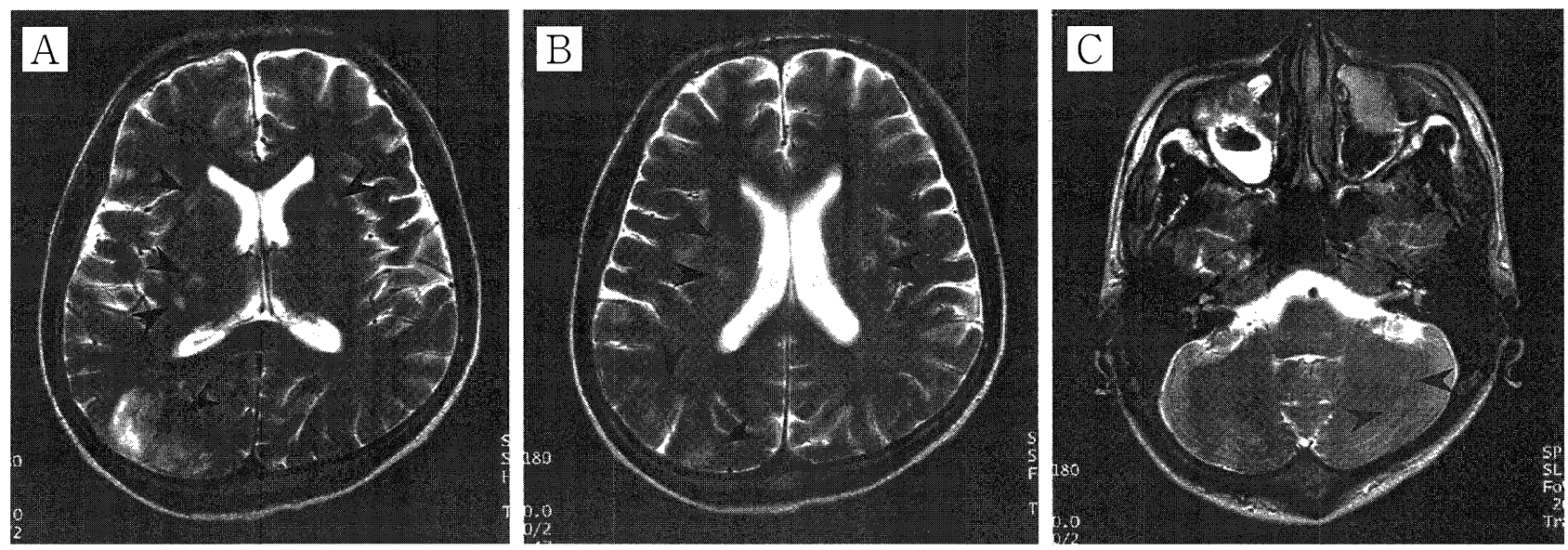

Figure 1. Cranial T2-weighted magnetic resonance imaging showed high intensity signals in the area extending between the right temporal lobe and occipital lobe $(\mathrm{A}, \mathrm{B})$, without a mass effect. Small lesions were also seen in basal ganglia bilaterally (A), deep white matter bilaterally (B) and left cerebellar hemisphere (C). The lesions are indicated by arrowheads.
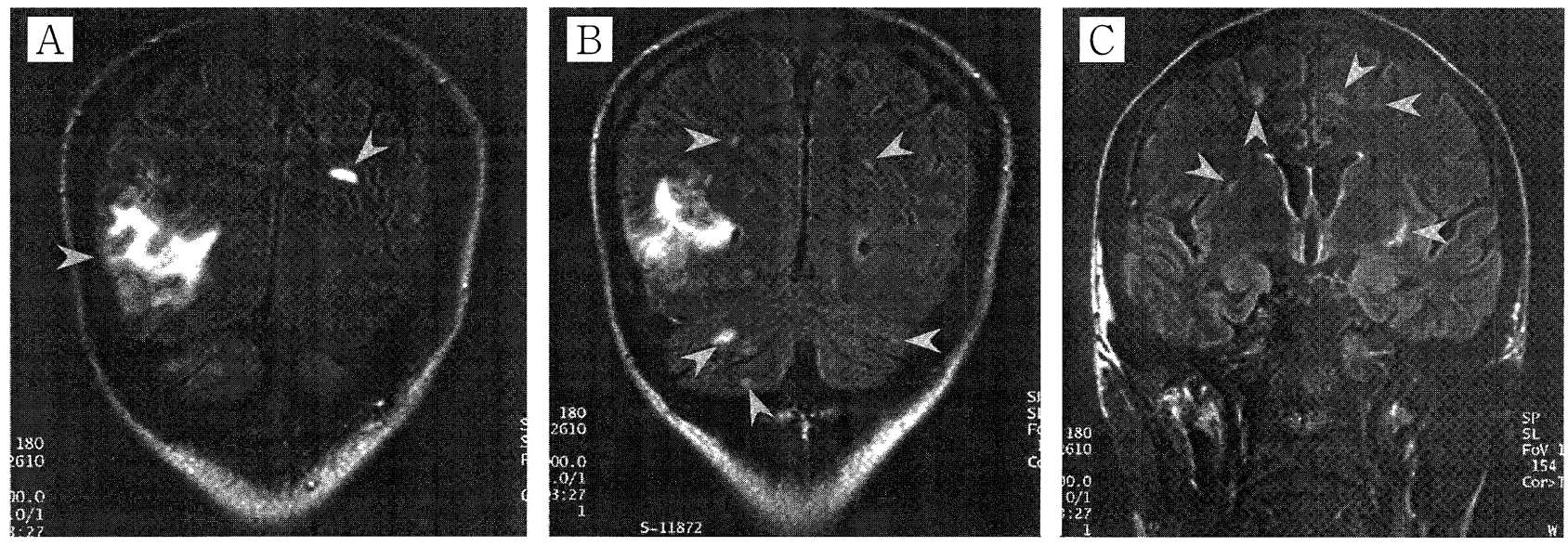

Figure 2. Fluid-attenuated inversion-recovery images (FLAIR) on coronal sections showed high intensity signals in the subcortical white matter between the right temporal lobe and occipital lobe (A, B), subcortical white matter of the parietal lobe (A, B, C), cerebellar hemisphere (B), and basal ganglia (C), bilaterally. The lesions are indicated by arrowheads. Abnormal signals in the subcortical region are more evident in FLAIR images than on T2-weighted images (compare with Fig. 1). 

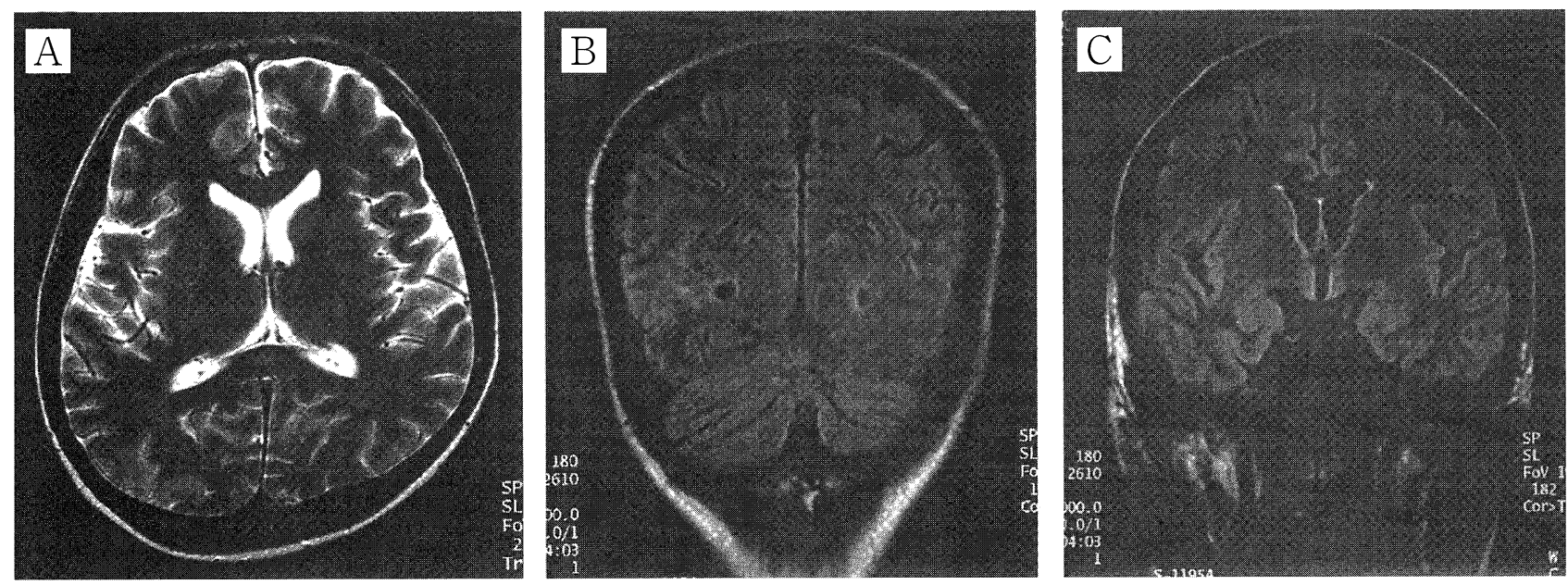

Figure 3. Repeat magnetic resonance imaging performed 14 days later showed almost complete resolution of abnormal areas on T2-weighted image (A) and FLAIR images (B, C).

Reversible posterior leukoencephalopathy syndrome is an uncommon neurological post-transplantation complication. It shows characteristic abnormalities on neuroimages, especially in the parieto-temporal-occipital region of the brain. Immunosuppressive agents, such as tacrolimus or cyclosporin, cause the development of this syndrome.

We report a 21-year-old man with myelodysplastic syndrome who had overt leukemia and underwent allogeneic stem cell transplantation. Tacrolimus was used to prevent acute graft versus host disease. At post-transplantation day 60, he developed seizure, cortical blindness, and disorientation. Cranial magnetic resonance imaging showed multiple abnormal signals in the area between the temporal lobe and occipital lobe as a main lesion, together with small abnormal signals in the parietal lobe, deep white matter, basal ganglia, and the cerebellum (Figs. 1 and 2). The patient showed complete recovery within two weeks after discontinuation of tacrolimus (Fig. 3).

This syndrome should be recognized, since it is reversible and readily controlled by stopping or reducing the immunosuppressive agent.

Tetsuro NAKAZATO, Akitoshi Nagasaki, Keita NAKAMURA,

Taeko OKUdAIRA, Aya OshiRo, Takashi MiYagi, Tetsuharu ShINJo,

Naoya TAIRA, Masato MASUDA and Nobuyuki TAKASU

The Second Department of Internal Medicine, Faculty of Medicine, The University of Ryukyus, Okinawa

Received for publication February 10, 2003; Accepted for publication March 31, 2003

Reprint requests should be addressed to Dr. Akitoshi Nagasaki, the Second Department of Internal Medicine,

Faculty of Medicine, The University of Ryukyus, 207 Uehara, Nishihara, Nakagami-gun, Okinawa 903-0215 